Keiko Sato · Mitsuru Emi - Yoichi Ezura $\cdot$ Yuko Fujita Daisuke Takada - Tomoaki Ishigami - Satoshi Umemura Yunpei Xin · Lily L. Wu $\cdot$ Stacey Larrinaga-Shum

Susan H. Stephenson · Steven C. Hunt

Paul N. Hopkins

\title{
Soluble epoxide hydrolase variant (Glu287Arg) modifies plasma total cholesterol and triglyceride phenotype in familial hypercholesterolemia: intrafamilial association study in an eight-generation hyperlipidemic kindred
}

Received: 25 August 2003 / Accepted: 16 October 2003/Published online: 13 December 2003

(C) The Japan Society of Human Genetics and Springer-Verlag 2003

\begin{abstract}
Plasma lipid and lipoprotein in general reflect the complex influences of multiple genetic loci, for instance, even familial hypercholesterolemia $(\mathrm{FH})$, a representative example of monogenic hyperlipidemia, often presents with phenotypic heterogeneity. In the course of investigating familial coronary artery disease in Utah, we studied 160 members of an eight-generation extended family of FH in which 69 members were affected with type IIa hyperlipoproteinemia (HLPIIa; high plasma cholesterol) and ten with type IIb hyperlipoproteinemia (HLPIIb; high plasma cholesterol as well as plasma triglyceride). Soluble epoxide hydrolase (EPHX2, sEH) plays a role in disposition of epoxides in plasma lipoprotein particles. Intrafamilial correlation analysis of the modifier effect of Glu287Arg substitution in the EPHX2 gene was carried out among $79 L D L R$ mutation carriers and 81 noncarriers. In the carriers, plasma cholesterol levels were elevated among carriers of the 287Arg allele (mean $\pm \mathrm{SD}=358 \pm 72 \mathrm{mg} / \mathrm{dl}$ ) in comparison with 287 Glu homozygotes (mean $\pm \mathrm{SD}=302 \pm 72 \mathrm{mg} / \mathrm{dl}$ ) $(p=0.0087)$. Similarly, in the $L D L R$ mutation carriers,
\end{abstract}

K. Sato $\cdot$ M. Emi $(\bowtie) \cdot Y$. Ezura $\cdot$ Y. Fujita $\cdot$ D. Takada Department of Molecular Biology, Institute of Gerontology, Nippon Medical School, 1-396 Kosugi-cho,

Kawasaki 211-8533, Japan

E-mail: memi@nms.ac.jp

Tel.: + 81-44-7335230

Fax: + 81-44-7335192

K. Sato $\cdot$ M. Emi $\cdot$ T. Ishigami $\cdot$ S. Umemura Department of Second Internal Medicine, Yokohama City University School of Medicine, Kanazawa-ku, Yokohama 236-0004, Japan

Y. Xin · L. L. Wu - S. Larrinaga-Shum · S. H. Stephenson · S. C. Hunt · P. N. Hopkins

Cardiovascular Genetics Research Clinic, University of Utah Hearth Science Center, 410 Chipeta Way, Salt Lake City, UT 84108, USA the plasma triglyceride levels were elevated among carriers of the 287Arg allele (mean $\pm \mathrm{SD}=260 \pm 100 \mathrm{mg} / \mathrm{dl}$ ) in comparison with $287 \mathrm{Glu}$ homozygotes (mean $\pm \mathrm{SD}=$ $169 \pm 83 \mathrm{mg} / \mathrm{dl})(p=0.020)$. No such gene-interactive effect was observed among noncarriers of the $L D L R$ mutation. Half of the patients who presented with HLPIIb had inherited a defective $L D L R$ allele as well as an EPHX2-287Arg allele, whereas the majority who presented with HLPIIa had a defective $L D L R$ allele but not an EPHX2-287Arg allele. These results indicate a significant modification of the phenotype of $\mathrm{FH}$ with defective $L D L R$ allele by $E P H X 2-287 \mathrm{Arg}$ variation in our studied kindred.

Keywords Soluble epoxide hydrolase $\cdot$ Familial hypercholesterolemia $\cdot$ Single nucleotide polymorphism (SNP) $\cdot$ Modifier gene $\cdot$ Triglyceride

\section{Introduction}

Hyperlipoproteinemia is one of the most important predictors of cardiovascular diseases determined by genetic risk factors as well as environmental factors (Hegele, 2001). Clarification of the genetic risk factors is essential for diagnosis, prevention, and early effective treatment of hyperlipidemias. The most prominent form of genetic hyperlipoproteinemia is familial hypercholesterolemia (FH), a common autosomal dominant disorder of lipoprotein metabolism with the prevalence of $1 / 500$ in the general population, and is at high risk of cardiovascular disease (Matsuzawa et al., 1995; Mabuchi et al., 1989). Defects in the gene coding LDL receptor $(L D L R)$ result in disturbed clearance of low-density lipoprotein cholesterol (LDL-C) because of impaired hepatic LDL receptor function. In heterozygous $\mathrm{FH}$ patients, an 
elevated level of the serum LDL-C is the characteristic criterion for diagnosis. FH patients often present with phenotypic heterogeneity, as plasma cholesterol levels can vary widely within the same pedigree; they may show increased plasma levels of triglyceride-rich lipoproteins in a variable spectrum that depends on segregation of other genetic loci. Potential influences of additional genetic factors on the variability and the severity of $\mathrm{FH}$, such as modifier genes interacting with the $L D L R$ mutation, have been assumed but rarely demonstrated (Emi et al., 1991; Hopkins et al., 1991).

In the course of investigating familial coronary artery disease in Utah, we ascertained an eight-generation FH pedigree consisting of 1,135 members; DNA were available from 160 members. This pedigree displayed varieties of clinical phenotypes and therefore provided an opportunity to dissect a variety of phenotype in FH by analyzing genetic variations at not only the $L D L R$ locus but also at loci of other genes modifying the phenotype. Accumulating evidences derived from clinical, epidemiological, and experimental studies suggest that phenotypic variation of human diseases reflect the influence of complex genetic loci, often referred to as modifier loci, a trait that is mostly traced by segregation of alleles defined by single nucleotide polymorphisms (SNPs) (Shastry et al., 2002). In the present study, we focused on analyzing the potential effect of SNP in the EPHX2 gene because it plays in disposition of epoxides in plasma lipoprotein particles.

\section{Materials and methods}

\section{Family and subjects}

A total of 160 members of a large FH pedigree (K653) were selected through the University of Utah Cardiovascular Genetics Research Clinic. This pedigree as a whole includes eight generations and 1,135 documented individuals; the roots of the pedigree were determined from genealogical records. The founding parents were born in the 1790s in the state of Vermont, USA; they were married in 1813 and had 12 children, but the offspring of only four of those children (a total of 80 grandchildren) are included for epidemiological studies. Molecular screening for $L D L R$ mutations in the members of $\mathrm{K} 653$ with clinically apparent $\mathrm{FH}$ led to identification of a novel splice-site mutation (IVS14+1 G $>$ A) shared by the members who were affected with elevated LDL-C. This mutation results in an abruptly truncated $L D L R$ protein, reducing functional $L D L R$ activity by half in heterozygous carriers of the mutant allele (Takada et al., 2002). We collected blood samples and isolated DNA in a manner previously described (Nakazawa et al., 2001; Iida et al., 2002). Among the 160 members tested, 79 carried the $L D L R$ heterozygous mutation and 81 did not. The study was approved by the Institutional Review Boards of the University of Utah and the Nippon Medical School, and all participants provided informed consent.

Measurement of lipoproteins

Fasting blood samples were obtained from 160 individuals in the pedigree with informed consent. After a $12-16 \mathrm{~h}$ fast, a blood sample was collected and prepared for each family member. If lipids from multiple blood draws were available, the lipoprotein profile from the first clinic visit was taken as the lipoprotein phenotype. Lipid and lipoprotein concentrations were measured by procedures described previously (Hattori et al., 2002). In brief, plasma cholesterol and triglyceride concentrations were assayed enzymatically, and concentrations of high-density lipoprotein cholesterol (HDL-C) were determined by the $\mathrm{MgCl}_{2}$ dextran precipitation method (Ishii et al., 2002). LDL was measured by ultracentrifugation after subtraction of HDL-C from HDL + LDL-C measured in the bottom fraction. These methods are routinely monitored and checked with both internal controls and standards supplied by the CDC Lipid Standardization Program.

Selection criteria of SNPs

We focused on all SNPs that accompany amino acid substitution (coding SNP, cSNP) and SNPs that locate in putative promoter region (regulatory SNP, rSNP) in the entire EPHX2 gene. The complete list of such SNPs are listed in Table 1 that describes nucleotide variation, amino acid variation, reference data base (JSNP and dbSNP), number of alleles in the studied Utah population and heterozygosity in that population. We examined SNPs whose heterozygosity exceeds 0.1 to obtain substantial statistic analytical power. Thus, out of the Q287R, S407I, and P550L listed in Table 1, Q287R was elected for the present study.

\section{Genotyping for SNPs in the EPHX2 gene}

PCR amplification of the polymorphism at the EPHX2 locus was performed using a condition described previously (Iida et al., 2002; Yoshida et al., 2002). Primer sequences used are as follows: Forward Primer: 5'-CCTGGCATTC TGCAGACGCTG-3', Reverse Primer: 5'-CTTGGAGCAT GAGCCTTAGGG -3'. The surrounding sequence of the amino acid-substituting SNP in the $E P H X 2$ gene, i.e., polymorphic nucleotides $\mathrm{A}$ or $\mathrm{G}$ in codons Glu287Arg (c $A \mathrm{~g} / \mathrm{cGg})$ as well as primer sequences and experimental condition were obtained from published reports (Haga et al., 2002). SNP genotyping was performed by Invader assay (Third Wave Technologies. Madison, WI, USA) (Saito et al., 2002) using PCR products of the flanking sequence and probes of the Invader assay designed and synthesized by the supplier (Ohnishi et al., 2001).

\section{Statistical analysis}

Plasma levels of lipoproteins were adjusted by gender and ages of the subjects. Coefficients of skewness and kurtosis were calculated to test a deviation from a normal distribution. Because the clinical and biochemical traits in each genotypic group did not always distribute normally, we applied the nonparametric Mann-Whitney test to compare those traits among groups divided by a single SNP (Fujiwara et al., 2002). Fisher's exact test was used to compare differences in the prevalence of type IIb hyperlipoproteinemia (HLPIIb) among the subgroups of the LDLR mutant carriers classified by relevant genotypes. Chi-square tests were invoked to detect Hardy-Weinberg equilibrium using the InStat 3 software package for Windows (GraphPad Software, San Diego, CA, USA).

\section{Results}

In the course of familial investigations of coronary artery disease, we identified an extended kinship consisting of 1,135 members, among whom a point mutation at the splice junction of intron 14 (IVS14+1 $\mathrm{G}>\mathrm{A}$ ) in the $L D L R$ gene was detected (Takada et al., 2002). Mutation screening of 160 members of an 
Table 1 Polymorphisms in the exons of the EPHX2 gene. SNP single nucleotide polymorphism

\begin{tabular}{lllllll}
\hline No & SNP & nt & Location & JSNP-ID $^{\mathrm{a}}$ & dbSNP $^{\mathrm{b}}$ & Number of alleles (heterozygosity) $^{\mathrm{c}}$ \\
\hline 1 & Q287R & A/G & Exon 8 & IMS-JST105448 & rs751141 & $135: 25(0.16)$ \\
2 & S407I & G/T & Exon 13 & IMS-JST105458 & rs4149251 & $159: 1(0.01)$ \\
3 & P550L & C/T & Exon 19 & - & rs4987060 & $158: 2(0.01)$ \\
\hline
\end{tabular}

${ }^{a}$ Number from Japanese SNP database (http://snp.ims.u-tokyo.ac.jp/index_ja.html)

${ }^{b}$ Number from dbSNP database of NCBI (http://www.ncbi.nlm.nih.gov/SNP/)

${ }^{c}$ Number of alleles $(n=160)$ from control individuals in Utah, USA

Table 2 Physical and clinical profiles of the subjects. Values are mean \pm SD. NS not significant

\begin{tabular}{|c|c|c|c|c|c|c|}
\hline & \multicolumn{3}{|c|}{ Carriers $(n=79)$} & \multicolumn{3}{|c|}{ Noncarriers $(n=81)$} \\
\hline Gender, F/M & $32 / 31$ & $10 / 6$ & NS & $40 / 32$ & $6 / 4$ & NS \\
\hline Age & $39.2 \pm 19.1$ & $35.2 \pm 20.3$ & NS & $40.0 \pm 22.2$ & $44.7 \pm 19.9$ & NS \\
\hline Total cholesterol $(\mathrm{mg} / \mathrm{dl})$ & $301.8 \pm 72.4$ & $358.3 \pm 72.1$ & 0.0087 & $195.2 \pm 40.9$ & $175.1 \pm 81.9$ & NS \\
\hline Triglyceride $(\mathrm{mg} / \mathrm{dl})$ & $168.7 \pm 82.5$ & $259.7 \pm 100.6$ & 0.020 & $149.0 \pm 80.9$ & $131.0 \pm 106.8$ & NS \\
\hline LDL-cholesterol (mg/dl) & $219.2 \pm 68.4$ & $252.4 \pm 85.9$ & NS & $118.9 \pm 34.4$ & $105.8 \pm 54.1$ & NS \\
\hline
\end{tabular}

${ }^{a} P$ values are for Mann-Whitney test except gender distribution. $\mathrm{Chi}^{2}$ test was applied for testing gender distribution

Fig. 1 Correlation between plasma total cholesterol level and the variation of the EPHX2 polymorphic nucleotides A or $\mathrm{G}$ in codons Glu287Arg (c $A \mathrm{~g}$ / $\mathrm{c} G \mathrm{~g}$ ) genotype. Total cholesterol level between two genotypic categories of Glu/Glu and Glu/Arg were analyzed both among $L D L R$ mutation carriers and among nonmutation carriers. Bars represent the mean values. Error bars indicate standard error $(S E)$. $P$ values are given by Mann-Whitney test. $n s$ not significant

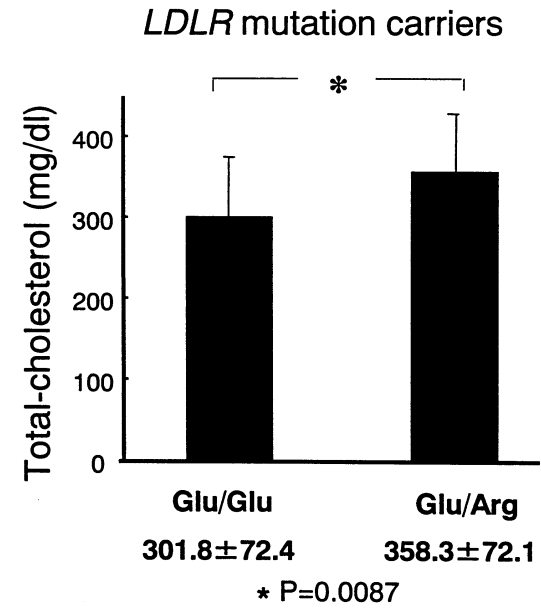

$L D L R$ mutation non-carriers

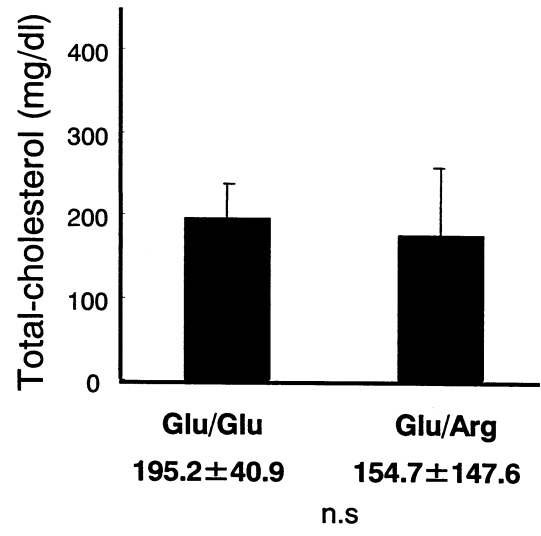

extended FH kinship (K653) segregating the splice junction mutation detected 79 heterozygous carriers and 81 noncarriers of $L D L R$ mutation among members whose genomic DNA was available.

As a part of a search for loci that might modify the defective $L D L R$ locus, we carried out an intrafamilial correlation analysis of the potential effect of SNPs in the $E P H X 2$ gene among $79 L D L R$ mutation carriers and 81 noncarriers in the test group from K653 (Table 2). We genotyped all subjects for the Glu287Arg variation (i.e., SNP; A or $\mathrm{G}$ in codon $\mathrm{c} A \mathrm{~g}$ or $\mathrm{c} G \mathrm{~g}$ substituting $287^{\text {th }} \mathrm{Glu}$ to Arg). No deviation of genotype frequencies was detected from the Hardy-Weinberg equilibrium; no 287Arg homozygous were present in our study subjects.

In the LDLR mutation carriers, the plasma cholesterol levels were elevated among carriers of the 287Arg allele (mean $\pm \mathrm{SD}=358 \pm 72 \mathrm{mg} / \mathrm{dl}$ ) in comparison with $287 \mathrm{Glu}$ homozygotes (mean $\pm \mathrm{SD}=302 \pm 72 \mathrm{mg} /$ dl) $(p=0.0087$, Mann-Whitney test) (Table 2, Fig. 1). Similarly, in the LDLR mutation carriers, the plasma triglyceride levels were elevated among carriers of 287Arg allele (mean $\pm \mathrm{SD}=260 \pm 101 \mathrm{mg} / \mathrm{dl}$ ) in comparison with $287 \mathrm{Glu}$ homozygotes (mean \pm $\mathrm{SD}=169 \pm 83 \mathrm{mg} / \mathrm{dl}) \quad(p=0.020) \quad$ (Table 2, Fig. 2). EPHX2-287Arg allele had similar elevating effects on LDL-C and VLDL-C, though it did not reach statistical significance. No such gene-interactive effect was observed among noncarriers of the LDLR mutation.

We classified the subjects into four genetic categories based on combinations of the LDLR mutation status and the EPHX2-287Glu/Arg variation status (Table 2). Mean plasma total-cholesterol levels in the two groups of LDLR mutation carriers were significantly higher than in the other two groups, as expected. In addition, 
Fig. 2 Correlation between plasma triglyceride level and the variation of the EPHX2 polymorphic nucleotides A or $\mathrm{G}$ in codons Glu287Arg (c $\mathrm{Ag}$ / $\mathrm{c} G \mathrm{~g}$ ) genotype. Triglyceride level between two genotypic categories of Glu/Glu and Glu/ Arg were analyzed both among $L D L R$ mutation carriers and among nonmutation carriers. Bars represent the mean values. Error bars indicate standard error $(S E)$. $P$ values are given by Mann-Whitney test. $n s$ not significant
$L D L R$ mutation carriers

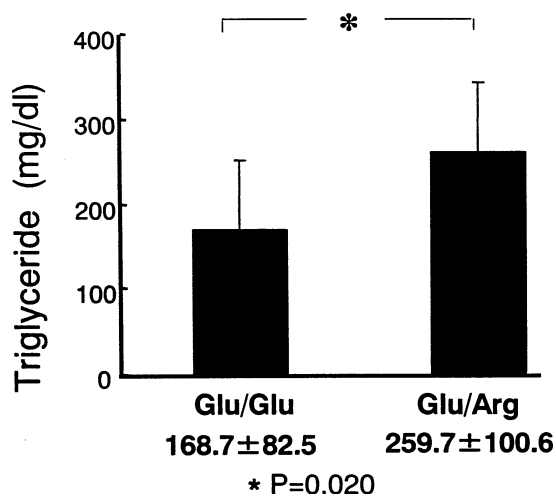

$L D L R$ mutation non-carriers

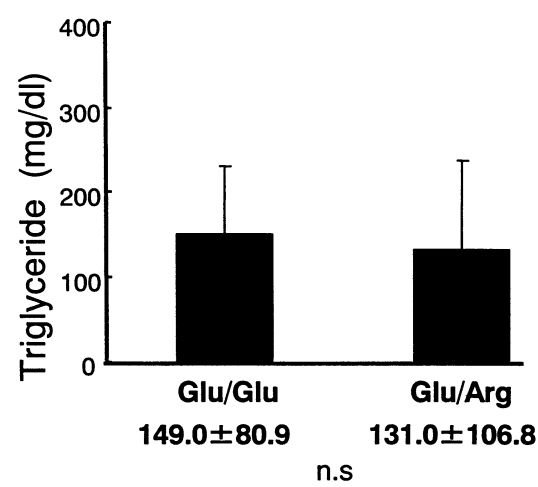

the group of 18 subjects who carried both mutant LDLR and EPHX2-287Arg alleles showed remarkably higher mean values for plasma triglyceride than the other three groups $(p=0.032)$. HDL-C levels were identical among all four groups. These data suggested an interactive effect of the EPHX2-287Arg allele as to elevation of plasma triglyceride levels among carriers of defective LDLR alleles. No modifying effect was observed among noncarriers of the LDLR mutation, and no significant differences in age or gender were observed among the three genotypic categories of LDLR mutation carriers.

Since the data presented here suggested involvement of at least two genetic loci, LDLR and EPHX2, in expression of the HLPIIb phenotype in this FH pedigree, we carried out a segregation analysis of members manifesting type IIa hyperlipoproteinemia (HLPIIa) and those manifesting HLPIIb with respect to inheritance of defective or normal alleles of the LDLR locus and $287 \mathrm{Glu}$ or $287 \mathrm{Arg}$ alleles of EPHX2 locus. A representative portion of the pedigree is shown in Fig. 3, in which lipoprotein phenotypes and genotypes for the LDLR and EPHX2 loci are symbolized for each member. HLPIIa was defined as an LDL-C level above the 90th percentile of reference values for age and gender (LDL-C > $141 \mathrm{mg} / \mathrm{dl}$ ) after age and gender adjustment; HLPIIb was defined as a phenotype of both LDL-C and triglyceride levels above the respective 90th percentiles (triglyceride $>288 \mathrm{mg} / \mathrm{dl}$ after age and gender adjustment) (Hegele et al., 2001). These criteria classified 69 carriers of the LDLR mutation as having HLPIIa and ten as having HLPIIb.

Half (5/10 cases) of the patients who presented with HLPIIb had inherited a defective $L D L R$ allele as well as an EPHX2-287Arg allele, whereas the majority (56/69 cases) who presented with HLPIIa had a defective $L D L R$ allele but not an EPHX2-287Glu allele.

\section{Discussion}

Phenotypic variation among FH patients appears to be influenced by multiple environmental and genetic factors (Hegele et al., 2001). Lifestyle variations among patients

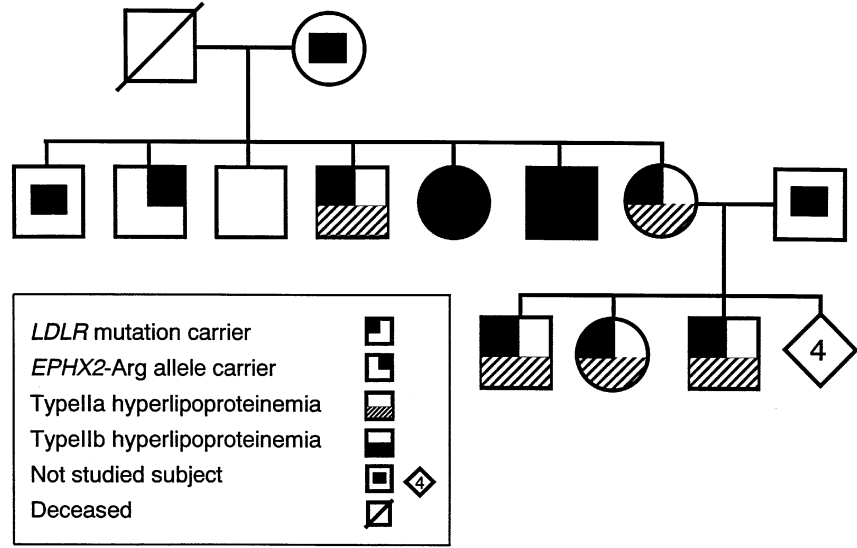

Fig. 3 Typical portion of pedigree K653, which consists of 1,135 members in all. Symbols (circles and sequences) filled in the upper left half indicate the $L D L R$ mutation carriers. Symbols filled in the upper right half indicate the EPHX2-287Arg allele carriers. Symbols filled with diagonal lines in the lower half indicate type IIa lipoprotein phenotype (HLPIIa). Symbols filled in the lower half indicate type IIb lipoprotein phenotype (HLPIIb)

in physical exercises, control of food calorie intake, psychological awareness of the disease, and compliance, including medications, must have influenced lipoprotein variations among individuals belonging to a specific genotype group. In addition, other unidentified genetic modifiers might have caused variability among the patients (Cullen et al., 1997). In addition to the primary physiologic effects of lipoprotein lipase (LPL) and cholesterol ester transfer protein (CETP), attention has been focused recently on the influence of these molecules on plasma lipoprotein levels in carriers of mutant LDLR alleles (Pimstone, et al., 1995, Haraki et al., 1997, Wittekoek et al., 1998). Moreover, variation in the microsomal triglyceride transfer protein gene (MTP) also influences lipoprotein phenotype of FH patients (Lundahl et al., 2000). These facts imply a complex etiology of hyperlipidemia involving heterogeneity at a variety of loci even in $\mathrm{FH}$ patients.

In the present study, we showed that a large portion of HLPIIb patients in an FH kindred had inherited variant alleles at two genetic loci, LDLR and EPHX2. 
An intrafamilial association study using 160 subjects from this large pedigree demonstrated an interaction between the defective $L D L R$ allele and a specific 287Arg allele in the EPHX2 gene, resulting in a marked increase in plasma cholesterol and triglycerides. The high prevalence of hypertriglyceridemia among $L D L R$ mutation carriers with EPHX2 287Arg alleles is in striking contrast to the scarceness of hypertriglyceridemia among the others. No such effect was observed among noncarriers of the $L D L R$ mutation, indicating an interacting effect of the $L D L R$ mutation and the EPHX2 variation.

We previously described a striking LDL-C-lowering effect of a defective apo B allele observed in LDLR carriers; we have previously described $L D L R$ mutation carriers in an FH pedigree who had normal cholesterol level with coinheritance of a truncated apo B (Emi et al., 1991). Moreover, we previously showed in four other extended FH pedigrees with complex lipoprotein phenotypes that elevated LDL-C concentrations also were due to defective LDLR genes, and that HLPIII occurred among individuals who had inherited a defective $L D L R$ allele along with a defective allele of the apolipoprotein E gene (apoE2). The latter mutation had a striking effect on remnant accumulation expressed as the VLDL cholesterol-to-triglyceride ratio in plasma, but only among individuals who also carried defective LDLR alleles (Emi et al., 1991; Hopkins et al., 1991). That study provided an early example of genetic interactions resulting in manifestation of a separate lipoprotein profile. More recently, we observed modification of the phenotype of FH by apo A-II gene variation (Takada et al., 2002).

Peroxisome proliferator-activated receptor $\gamma(\operatorname{PPAR} \gamma)$ plays central roles in lipid metabolism and glucose homeostasis; 15 -deoxy- $\Delta^{12,14}$ - prostaglandin $\mathrm{J}_{2}$ (15d$\mathrm{PGJ}_{2}$ ), is its endogenous ligand and is a derivative of epoxides that include arachidonic acids, eicosapentaenoic acids, and epoxyeicosatrienoic acids (EETs) (Rosen et al., 1999). These bioactive molecules are hydroxylated by soluble epoxide hydrolase (sEH) in liver, an enzyme directing the functional disposition of a variety of epoxides. Thus, the pharmacological attenuation of sEH activity causes secondary increase in epoxide levels as well as variety of subsequent reactions. The $15 \mathrm{~d}-\mathrm{PGJ}_{2}$, being a high-affinity ligand of PPAR $\gamma$, is essential for adipocyte differentiation and lipid metabolism through leptin (Hollenberg et al., 1997). Variation of sEH activity may modify plasma $15 \mathrm{~d}-\mathrm{PGJ}_{2}$ levels and affect lipid metabolism through PPAR $\gamma$ activation. Another type of EET, eicosapentaenoic acid, regulates triglyceride synthesis (Wang et al., 1993, Kurokawa et al., 1995, Rustan et al., 1988). Relationships between these epoxides and the lipoprotein phenotype of patients should be clarified in the future study. The EPHX2 gene is expressed predominantly in the liver, intestine, and kidney and at minimal levels in heart and testis, lung, brain, and spleen in humans. Although we aimed to carry out enzymatic and protein mass assay for EPHX2 protein in the population, we were unable to do such analyses because biopsy specimens of liver, intestine, or kidney were unavailable from family members of the present kindred. The EPHX2 Glu287Arg variation itself may have direct effect in the EPHX2 function; however, the possibility of the presence of other unidentified functional variants in linkage disequilibrium can not be ruled out at present, as well as the effect of variations in other yet unidentified gene adjacent to the EPHX2 gene.

In summary, we identified a novel gene-gene interaction influencing clinical phenotype of $\mathrm{FH}$ by investigating a large family for molecular defects at two loci. HapMap initiative is now in progress among the international consortium. Haplotypic knowledge of the human genome will help clarify the contribution of the $E P H X 2$ gene in lipid profile through detailed haplotype association study in the near future. Our study illustrates the feasibility of analyzing interactions among multiple loci as an approach to the etiology of complex metabolic diseases. Indeed, we expect that the effects of multiple genes, both additive and interactive, will eventually prove to be responsible for many cases of common, inherited, mixed dyslipidemias (Lalouel et al., 2001). Further study will lead to better understandings of regulatory systems of plasma lipid metabolism and identification of new investigation targets in the treatment of hyperlipidemic patients.

Acknowledgements We thank Mitsuko Kajita, Mina Kodaira, Miho Kawagoe, Kyoko Shimizu, Mayumi Tanaka, and Naoko Tsuruta for their expert technical assistance. This work was supported by a grant for Strategic Research from the Ministry of Education, Science, Sports and Culture of Japan; by a Research Grant for Research from the Ministry of Health and Welfare of Japan; and by a Research for the Future Program Grant of The Japan Society for the Promotion of Science.

\section{References}

Cullen P, Funke H, Schulte H, Assmann G (1997) Lipoproteins and cardiovascular risk-from genetics to $\mathrm{CHD}$ prevention. J Atheroscler Thromb 4:51-58

Emi M, Hegele RM, Hopkins PN, Wu LL, Plaetke R, Williams RR, Lalouel JM (1991) Effects of three genetic loci in a pedigree with multiple lipoprotein phenotypes. J Arterioscler Thromb 11:1349-1355

Fujiwara H, Emi M, Nagai H, Nishimura T, Konishi N, Kubota Y, Ichikawa T, Takahashi S, Shuin T, Habuchi T, Ogawa O, Inoue K, Skolnick MH, Swensen J, Camp NJ, Tavtigian SV (2002) Association of common missense changes in ELAC2 (HPC2) with prostate cancer in a Japanese case-control series. J Hum Genet 47:641-648

Haga H, Yamada R, Ohnishi, Y, Nakamura Y, Tanaka T (2002) Gene-based SNP discovery as part of the Japanese Millennium Genome Project: identification of 190, 562 genetic variations in the human genome. J Hum Genet 47:605-610

Haraki T, Inazu A, Yagi K, Kajinami K, Koizumi J, Mabuchi H (1997) Clinical characteristics of double heterozygous with familial hypercholesterolemia and cholesteryl ester transfer protein deficiency. Atherosclerosis 132:229-236

Hattori H, Hirayama T, Nobe Y, Nagano M, Kujiraoka T, Egashira T, Ishii J, Tsuji M, Emi M (2002) Eight novel mutations and functional impairments of the LDL receptor in familial hypercholesterolemia in the north of Japan. J Hum Genet 47:80-87 
Hegele RA (2001) Monogenic dyslipidemias: window on determinants of plasma lipoprotein metabolism. Am J Hum Genet 69:1161-1177

Hollenberg AN, Susulic VS, Madura JP, Zhang B, Moller DE, Tontonoz P, Sarraf P, Spiegelman BM, Lowell BB (1997) Functional antagonism between CCAAT/enhancer binding protein-alpha and peroxisome proliferator-activated receptorgamma on the leptin promoter. J Biol Chem 272:5283-5290

Hopkins PN, Wu LL, Schumacher MC, Emi M, Hegele RM, Hunt SC, Lalouel JM, Williams RR (1991) Type III dyslipoproteinemia in patients heterozygous for familial hypercholesterolemia and apolipoprotein E2 Evidence for a gene-gene interaction. ArteriosclerThromb 11:1137-1146

Iida A, Saito S, Sekine A, Mishima C, Kitamura Y, Kondo K, Harigae S, Osawa S, Nakamura Y (2002) Catalog of 77 singlenucleotide polymorphisms (SNPs) in the carbohydrate sulfotransferase 1 (CHST1) and carbohydrate sulfotransferase 3 (CHST3) genes. J Hum Genet 47; 14-19

Ishii J, Nagano M, Kujiraoka T, Ishihara M, Egashira T, Takada D, Tsuji M, Hattori H, Emi M (2002) Clinical variant of Tangier disease in Japan: mutation of the ABCA1 gene in hypoalphalipoproteinemia with corneal lipidosis. J Hum Genet 47:366-369

Kurokawa M, Hirano T, Furukawa S, Nagano S, Adachi M (1995) Similar to oleic acid, eicosapentaenoic acid stimulates apolipoprotein B secretion by inhibiting its intracellular degradation in Hep G2 cells Atherosclerosis 112:59-68

Lalouel JM, Rohrwasser A (2001) Development of genetic hypotheses in essential hypertension. J Hum Genet 46:299-306

Lundahl B, Leren TP, Ose L, Hamsen A, Karpe F (2000) A functional polymorphism in the promoter region of the microsomal triglyceride transfer protein (MTP-493G/T) influences lipoprotein phenotype in familial hypercholesterolemia. Atheroscler Thromb Vasc Biol 20:1784-1788

Mabuchi H, Koizumi J, Shimizu M, Takeda R (1989) Development of coronary heart disease in familial hypercholesterolemia. Circulation 79:225-232

Matsuzawa Y (1995) Significance of hyperglycemia in the occurrence of ischemic heart disease. J Atheroscler Thromb 2:S26-28

Nakazawa I, Nakajima T, Ishigami T, Umemura S, Emi M (2001) Linkage disequilibrium and haplotype analysis among eight novel single-nucleotide polymorphisms in the human tissuetype plasminogen activator (t-PA) gene. J Hum Genet 46:367-371.
Ohnishi Y, Tanaka T, Ozaki K, Yamada R, Suzuki, H, Nakamura Y (2001) A high-throughput SNP typing system for genomewide association studies. J Hum Genet 46:471-477

Pimstone SN, Gagne SE, Gagne C, Lupien PJ, Gaudet D, Williams RR, Kotze M, Reymer PW, Defesche JC, Kastelen JJ (1995) Mutations in the gene for lipoprotein lipase A cause for low HDL cholesterol levels in individuals heterozygous for familial hypercholesterolemia. Arterioscler Thromb Vasc Biol 15:17041712

Rosen ED, Sarraf P, Troy AE, Bradwin G, Moore K, Milstone DS, Spiegelman BM, Mortensen RM (1999) PPAR gamma is required for the differentiation of adipose tissue in vivo and in vitro. Mol Cell 4:611-617

Rustan AC, Nossen JO, Christiansen EN, Drevon CA (1988) Eicosapentaenoic acid reduces hepatic synthesis and secretion of triacylglycerol by decreasing the activity of acyl-coenzyme A: 1,2-diacylglycerol acyltransferase. J Lipid Res 29:1417-1426

Saito S, Iida A, Sekine A, Miura Y, Ogawa C, Kawauchi S, Higuchi S, Nakamura Y (2002) Identification of 779 genetic variations in eight genes encoding members of the ATP-binding cassette, subfamily C (ABCC/MRP/CFTR) J Hum Genet 47:147-171

Shastry BS (2002) Minireview: SNP alleles in human disease and evolution. J Hum Genet 47:561-566

Takada D, Emi M, Ezura Y, Nobe Y, Kawamura K, Iino Y, Katayama Y, Xin Y, Wu LL, Shum SL, Stephenson SH, Hunt SC, Hopkins PN (2002) Interaction between the LDL-receptor gene bearing a novel mutation and a variant in the apolipoprotein A-II promoter: molecular study in a 1135-member familial hypercholesterolemia kindred. J Hum Genet 47:656664

Wang H, Chen X, Fisher EA (1993) N-3 fatty acids stimulate intracellular degradation of apolipoprotein $\mathrm{B}$ in rat hepatocytes. J Clin Invest 91:1380-1389

Wittekoek ME, Pimstone SN, Reymer PW, Feuth L, Botma GJ, Defesche JC, Prins M, Hayden MR, Kastelein JJ (1998) A common mutation in the lipoprotein lipase gene (N291S) alters the lipoprotein phenotype and risk for cardiovascular disease in patients with familial hypercholesterolemia. Circulation 97:729-735

Yoshida S, Harada H, Nagai H, Fukino K, Teramoto A, Emi M (2002) Head-to-head juxtaposition of Fas-associated phosphatase-1 (FAP-1) and c-Jun $\mathrm{NH}_{2}$-terminal kinase 3 (JNK3) genes: genomic structure and seven polymorphisms of the FAP-1 gene. J Hum Genet 47:614-619 